Supporting information

\title{
In vivo activity of repurposed amodiaquine as a host-targeting therapy for the treatment of anthrax.
}

Mikhail Martchenko Shilman ${ }^{1,2, *}$, Gloria Bartolo ${ }^{1}$, Saleem Alameh ${ }^{1}$, Johnny W. Peterson ${ }^{3}$, William S. Lawrence ${ }^{3}$, Jennifer E. Peel ${ }^{3}$, Satheesh K. Sivasubramani ${ }^{4}$, David W.C. Beasley ${ }^{3}$, Christopher K. Cote ${ }^{5}$, Samandra T. Demons ${ }^{5}$, Stephanie A. Halasahoris ${ }^{5}$, Lynda L. Miller ${ }^{5}$, Christopher P. Klimko ${ }^{5}$, Jennifer L. Shoe ${ }^{5}$, David P. Fetterer ${ }^{6}$, Ryan McComb ${ }^{1}$, Chi-Lee C. Ho ${ }^{7}$, Kenneth A. Bradley ${ }^{7}$, Stella Hartmann ${ }^{1}$, Luisa W. Cheng $^{8}$, Marina Chugunova ${ }^{9}$, Chiu-Yen Kao ${ }^{10}$, Jennifer K. Tran ${ }^{1}$, Aram Derbedrossian ${ }^{1}$, Leeor Zilbermintz ${ }^{1}$, Emiene Amali-Adekwu ${ }^{1}$, Anastasia Levitin $^{1}$, and Joel West ${ }^{1,2}$

${ }^{1}$ Henry E. Riggs School of Applied Life Sciences, Keck Graduate Institute (KGI), 535 Watson Drive, Claremont, CA 91711

${ }^{2}$ Shield Pharma LLC, 1420 N Claremont Blvd Suite 102A, Claremont, CA 91711

${ }^{3}$ Department of Microbiology and Immunology, University of Texas Medical Branch (UTMB), 301 University Blvd, Galveston, TX 77555

${ }^{4}$ Directorate of Environmental Health Effects Laboratory, Naval Medical Research Unit, 2728 Q Street Bldg 837, Wright-Patterson AFB, OH 45433

${ }^{5}$ Bacteriology Division, US Army Medical Research Institute of Infectious Diseases (USAMRIID), 1425 Porter St, Fort Detrick, Frederick, MD 21702 
${ }^{6}$ Biostatistics Division, US Army Medical Research Institute of Infectious Diseases

(USAMRIID), 1425 Porter St, Fort Detrick, Frederick, MD 21702

${ }^{7}$ Department of Microbiology, Immunology and Molecular Genetics, University of California, Los Angeles (UCLA), 609 Charles E. Young Drive East, Los Angeles, CA 90095

${ }^{8}$ Foodborne Toxin Detection and Prevention Research Unit, Western Regional Research Center, United States Department of Agriculture (USDA), 800 Buchanan Street, Albany, CA 94710

${ }^{9}$ Institute of Mathematical Sciences, Claremont Graduate University (CGU), 150 E. 10th Str., Claremont, CA 91711

${ }^{10}$ Department of Mathematical Sciences, Claremont McKenna College (CMC), 888 N Columbia Ave, Claremont, CA 91711

* corresponding authors mikhail_shilman@kgi.edu

Contents (20 pages)

Figure S1: Weights of anthrax-infected rabbits receiving varying doses of AQ.

Figure S2: Weights of anthrax-infected rabbits receiving levofloxacin and AQ.

Figure S3: Weights of anthrax-infected rabbits receiving levofloxacin and delayed AQ treatment.

Table S1: Clinical observations of individual rabbits after intravenous administration of AQ and DEAQ.

Table S2: Clinical observations of individual rabbits after oral administration of AQ.

Table S3: Pharmacokinetics of individual rabbits after intravenous administration of AQ and DEAQ. 
Table S4: Pharmacokinetics of individual rabbits after oral administration of AQ.

Table S5: Therapeutic dosing of AQ and blood collection schedule of anthrax-infected rabbits.

Table S6: Anthrax-related events of individual rabbits after oral administration of AQ.

Table S7: Therapeutic dosing and blood collection schedule of anthrax-infected rabbits receiving levofloxacin and AQ.

Table S8: Anthrax-related events of individual rabbits after oral administration of AQ.

Table S9: Therapeutic dosing and blood collection schedule of anthrax-infected rabbits receiving levofloxacin and delayed AQ treatment.

Table S10: Identification of side effects in AQ safety articles via Python.

Table S11: Identification of side effects in AQ safety articles via Matlab.

Table S12: Identification of side effects in AQ articles via Python.

Table S13: Identification of side effects in AQ articles via Matlab. 


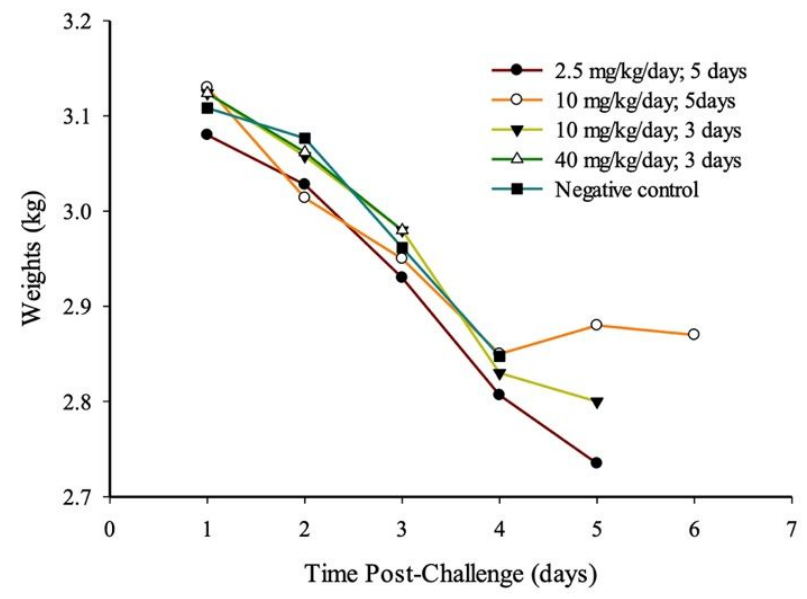

Figure S1: Weights of anthrax-infected rabbits receiving varying doses of $A Q$.

Weights of anthrax-infected NZW rabbits were measured in the absence or presence of varying doses of AQ administered for either 3 or 5 days. Animal weights were monitored once daily for as long as the animal lived. Treatment groups consisted of 5 rabbits each, while the control group consisted of 6 rabbits. 

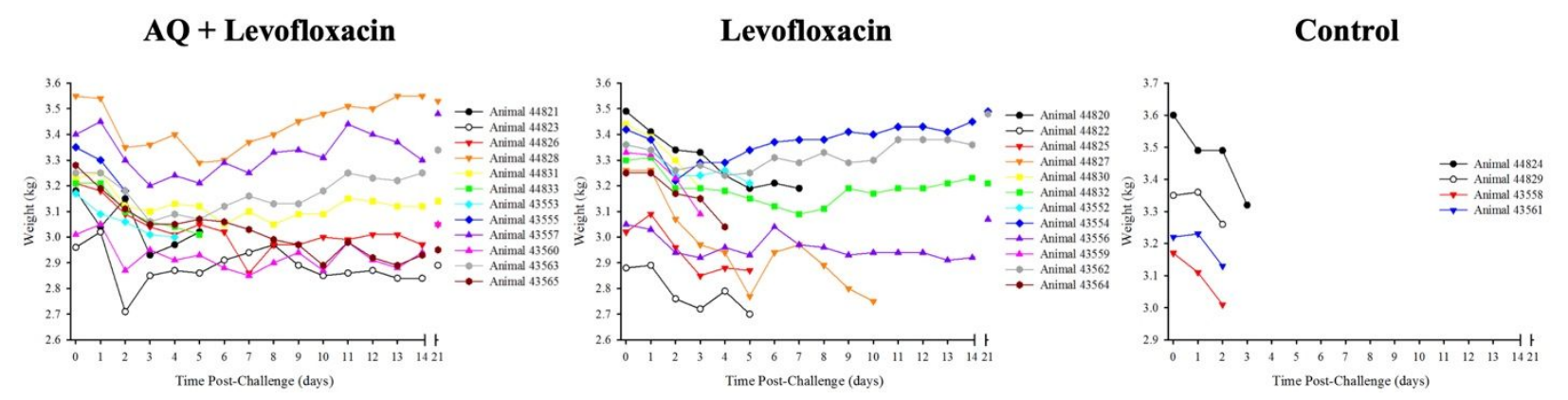

Figure S2: Weights of anthrax-infected rabbits receiving levofloxacin and AQ.

Weights of anthrax-infected NZW rabbits were measured for animals administered suboptimal dose $(1.6 \mathrm{mg} / \mathrm{kg} /$ day) of levofloxacin (middle panel) or with $10 \mathrm{mg} / \mathrm{kg} /$ day of AQ in addition to levofloxacin (left panel). Animal weights for control rabbits were also measured (right panel). Animal weights were monitored once daily for as long as the animal lived. Treatment groups consisted of 12 rabbits each, while the control group consisted of 4 rabbits. 

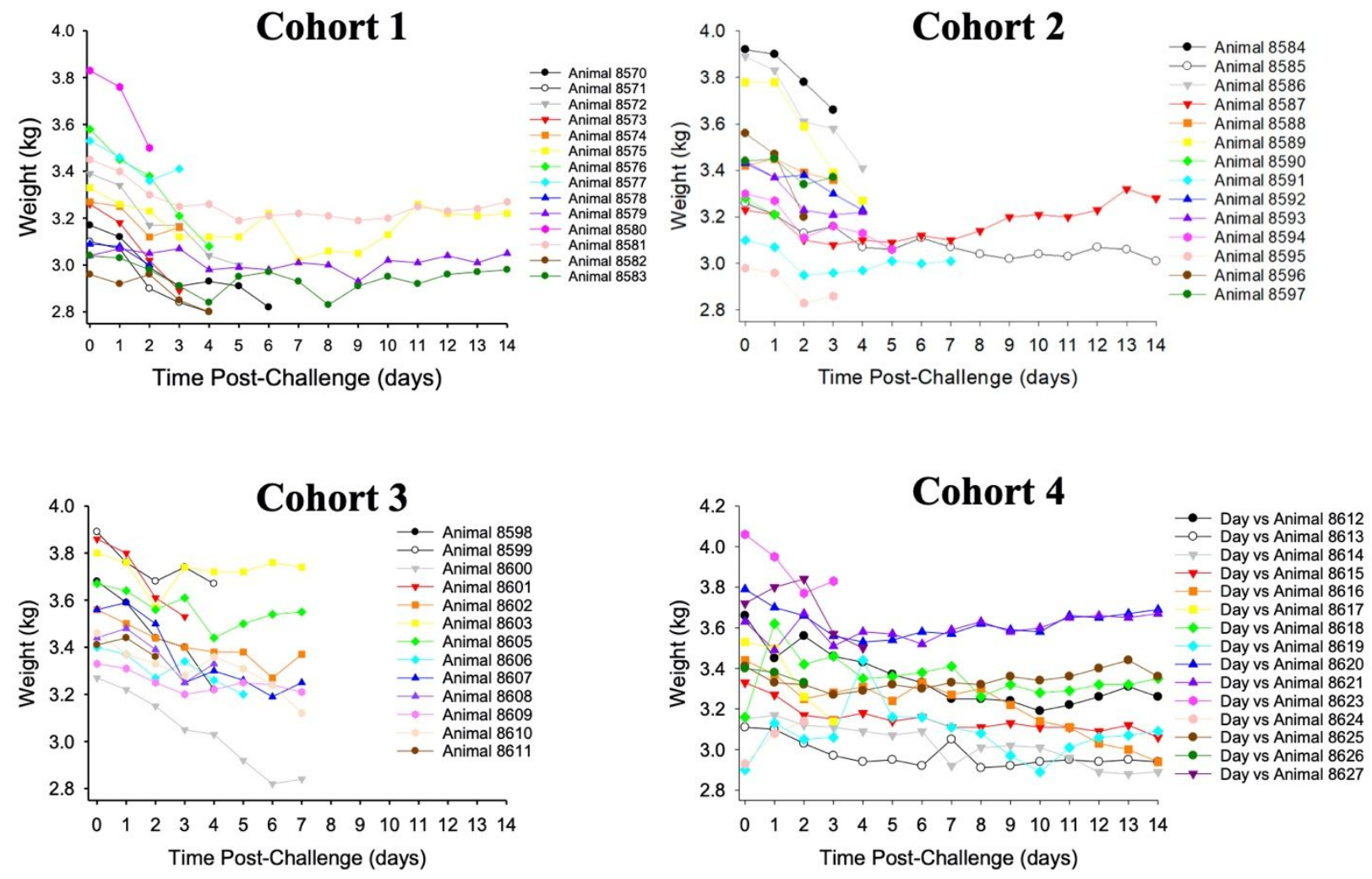

Figure S3: Weights of anthrax-infected rabbits receiving levofloxacin and delayed AQ treatment.

Weights of all anthrax-infected NZW rabbits were measured throughout the study. Due to the large study size, animals were divided into four cohorts. Each cohort included at least one animal from each group. Animal weights were monitored once daily for as long as the animal lived. 


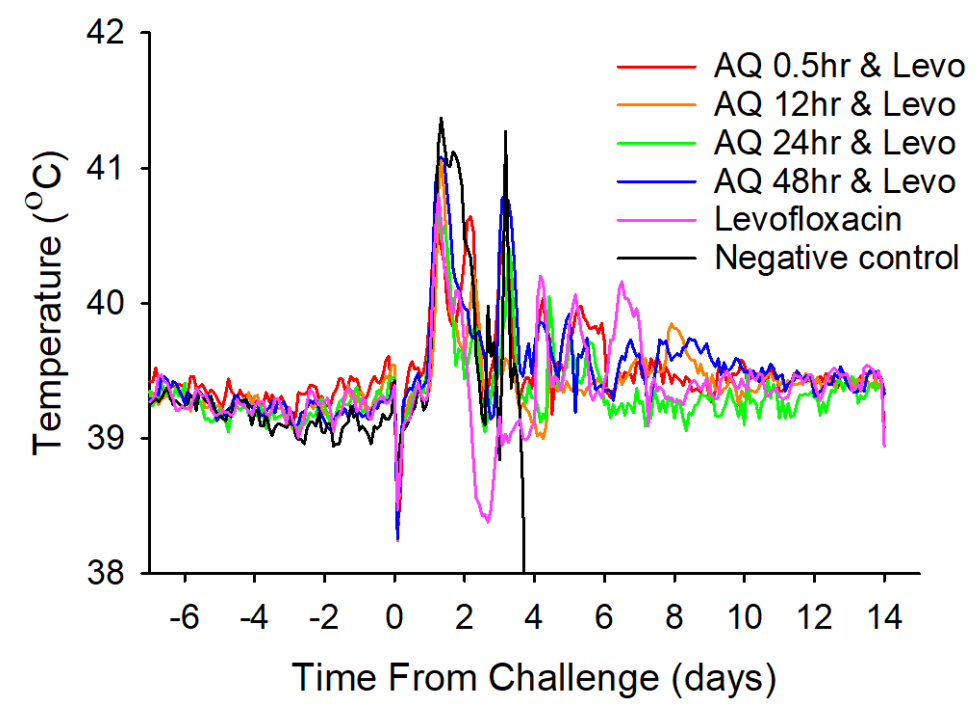

Figure S4: Temperature of anthrax-infected rabbits receiving levofloxacin and delayed AQ treatment.

The average core body temperatures of the treatment groups are presented as two-hour moving averages. 
Table S1: Clinical observations of individual rabbits after intravenous administration of AQ

and DEAQ.

\begin{tabular}{|c|c|c|c|c|c|c|c|}
\hline \multirow[b]{3}{*}{ Drug } & \multirow[b]{3}{*}{ Sex } & \multirow[b]{3}{*}{ Animal } & \multirow{3}{*}{ Clinical Sign } & & \multicolumn{3}{|c|}{$\begin{array}{l}\text { Number of days } \\
\text { relative to start date }\end{array}$} \\
\hline & & & & & 1 & 2 & 3 \\
\hline & & & & Site & & & \\
\hline \multirow{19}{*}{ AQ - intravenous } & \multirow{6}{*}{ Male } & \multirow{2}{*}{001} & No abnormalities detected & & $\mathrm{X}$ & $\mathrm{X}$ & $\mathrm{X}$ \\
\hline & & & Main sacrifice & & $\cdot$ & $\cdot$ & $\mathrm{X}$ \\
\hline & & \multirow{2}{*}{002} & No abnormalities detected & & $\mathrm{X}$ & $\mathrm{X}$ & $\mathrm{X}$ \\
\hline & & & Main sacrifice & & $\cdot$ & $\cdot$ & $\mathrm{X}$ \\
\hline & & \multirow{2}{*}{003} & No abnormalities detected & & $\mathrm{X}$ & $\mathrm{X}$ & $\mathrm{X}$ \\
\hline & & & Main sacrifice & & $\cdot$ & $\cdot$ & $\mathrm{X}$ \\
\hline & \multirow{13}{*}{ Female } & \multirow{2}{*}{$104 \dagger$} & No abnormalities detected & & $\mathrm{X}$ & $\mathrm{X}$ & $\mathrm{X}$ \\
\hline & & & Main sacrifice & & $\cdot$ & $\cdot$ & $X$ \\
\hline & & \multirow{7}{*}{005} & Ataxia & & $\mathrm{S}$ & . & $\cdot$ \\
\hline & & & Convulsion & & E & $\cdot$ & $\cdot$ \\
\hline & & & Nystagmus & Left & $\mathrm{S}$ & $\cdot$ & $\cdot$ \\
\hline & & & Tachypnea & & M & $\cdot$ & $\cdot$ \\
\hline & & & Tremors & & $\mathrm{E}$ & $\cdot$ & $\cdot$ \\
\hline & & & Weakness & & $\mathrm{S}$ & $\cdot$ & $\cdot$ \\
\hline & & & Removed from Study & & $X$ & . & $\cdot$ \\
\hline & & \multirow{4}{*}{006} & Convulsion & & $\mathrm{E}$ & $\cdot$ & $\cdot$ \\
\hline & & & Hypoactivity $\ddagger$ & & $\mathrm{S}$ & $\cdot$ & $\cdot$ \\
\hline & & & Tachypnea & & M & . & $\cdot$ \\
\hline & & & Removed from Study & & $\mathrm{X}$ & $\cdot$ & $\cdot$ \\
\hline \multirow{13}{*}{ DEAQ - intravenous } & \multirow{6}{*}{ Male } & \multirow{2}{*}{007} & No abnormalities detected & & $\mathrm{X}$ & $\mathrm{X}$ & $\mathrm{X}$ \\
\hline & & & Main sacrifice & & $\cdot$ & . & $X$ \\
\hline & & \multirow{2}{*}{008} & No abnormalities detected & & $\mathrm{X}$ & $\mathrm{X}$ & $\mathrm{X}$ \\
\hline & & & Main sacrifice & & $\cdot$ & $\cdot$ & $\mathrm{X}$ \\
\hline & & \multirow{2}{*}{009} & No abnormalities detected & & $\mathrm{X}$ & $\mathrm{X}$ & $X$ \\
\hline & & & Main sacrifice & & $\cdot$ & $\cdot$ & $\mathrm{X}$ \\
\hline & \multirow{7}{*}{ Female } & \multirow{2}{*}{010} & No abnormalities detected & & $\mathrm{X}$ & $\mathrm{X}$ & $\mathrm{X}$ \\
\hline & & & Main sacrifice & & $\cdot$ & . & $\mathrm{X}$ \\
\hline & & \multirow{3}{*}{011} & No abnormalities detected & & $\mathrm{X}$ & $\mathrm{X}$ & $\mathrm{X}$ \\
\hline & & & Hypoactivity & & $\mathrm{S}$ & $\cdot$ & $\cdot$ \\
\hline & & & Main sacrifice & & $\cdot$ & $\cdot$ & $\mathrm{X}$ \\
\hline & & \multirow{2}{*}{012} & No abnormalities detected & & $\mathrm{X}$ & $\mathrm{X}$ & $\mathrm{X}$ \\
\hline & & & Main sacrifice & & $\cdot$ & . & $\mathrm{X}$ \\
\hline
\end{tabular}

$\uparrow$ Replacement animal. Female rabbit (004) died immediately post-dose.

$\$$ Hypoactivity increased in severity (to extreme) after a few minutes when the other signs showed.

Clinical observations of individual male and female NZW rabbits were monitored after animals were given a single dose of $10 \mathrm{mg} / \mathrm{kg}$ of either AQ or DEAQ intravenously. Clinical signs were documented on the day of occurrence, the severity of clinical sign(s), and site of clinical sign(s), 
if available. The animals were monitored for three days post-dose, if not removed from the study prior. 
Table S2: Clinical observations of individual rabbits after oral administration of AQ.

\begin{tabular}{|c|c|c|c|c|c|c|c|}
\hline & & & & & $\begin{array}{r}\text { Nur } \\
\text { relati }\end{array}$ & $\begin{array}{l}\text { er o } \\
\text { o st }\end{array}$ & $\begin{array}{l}\text { ys } \\
\text { date }\end{array}$ \\
\hline & & & & & 1 & 2 & 3 \\
\hline Drug & Sex & Animal & Clinical Sign & Site & & & \\
\hline \multirow{15}{*}{$\mathrm{AQ}$ - oral } & \multirow{7}{*}{ Male } & \multirow{2}{*}{001} & No abnormalities detected & & $\mathrm{X}$ & $\mathrm{X}$ & $\mathrm{X}$ \\
\hline & & & Main sacrifice & & . & . & $\mathrm{X}$ \\
\hline & & \multirow{3}{*}{002} & No abnormalities detected & & $\mathrm{X}$ & . & $\mathrm{X}$ \\
\hline & & & Few feces & & . & $\mathrm{S}$ & . \\
\hline & & & Main sacrifice & & . & . & $\mathrm{X}$ \\
\hline & & \multirow{2}{*}{003} & No abnormalities detected & & $\mathrm{X}$ & $\mathrm{X}$ & $\mathrm{X}$ \\
\hline & & & Main sacrifice & & . & . & $\mathrm{X}$ \\
\hline & \multirow{8}{*}{ Female } & \multirow{2}{*}{004} & No abnormalities detected & & $\mathrm{X}$ & $\mathrm{X}$ & $\mathrm{X}$ \\
\hline & & & Main sacrifice & & . & . & $\mathrm{X}$ \\
\hline & & \multirow{2}{*}{005} & No abnormalities detected & & $\mathrm{X}$ & $\mathrm{X}$ & $\mathrm{X}$ \\
\hline & & & Main sacrifice & & . & . & $\mathrm{X}$ \\
\hline & & \multirow{4}{*}{006} & No abnormalities detected & & $\mathrm{X}$ & . & . \\
\hline & & & Few feces & & . & $\mathrm{M}$ & M \\
\hline & & & Reduced appetite & & . & $\mathrm{X}$ & $\mathrm{X}$ \\
\hline & & & Main sacrifice & & . & . & $\mathrm{X}$ \\
\hline
\end{tabular}

Clinical observations of individual male and female NZW rabbits were monitored after animals were given a single dose of $10 \mathrm{mg} / \mathrm{kg}$ of AQ orally. Clinical signs were documented on the day of occurrence, the severity of clinical sign(s), and site of clinical sign(s), if available. The animals were monitored for three days post-dose. 
Table S3: Pharmacokinetics of individual rabbits after intravenous administration of AQ

and DEAQ.

\begin{tabular}{|c|c|c|c|c|c|c|c|c|c|c|}
\hline Drug & Sex & Animal & Analyte & $\begin{array}{l}\mathbf{T}_{\max } \\
\text { (hr) }\end{array}$ & $\begin{array}{l}C_{\max } \\
(\mu \mathrm{M})\end{array}$ & $\begin{array}{c}\mathbf{t}_{1 / 2} \\
(\mathbf{h r})\end{array}$ & $\begin{array}{c}\mathbf{A U} \mathbf{C}_{\text {last }} \\
(\mathbf{h r} \cdot \boldsymbol{\mu} \mathbf{M})\end{array}$ & $\begin{array}{c}A U C_{\text {inf }} \\
(\mathbf{h r} \cdot \mu M)\end{array}$ & $\begin{array}{c}\mathrm{Cl} \\
(\mathrm{ml} / \mathrm{hr} / \mathbf{k g})\end{array}$ & $\begin{array}{c}\mathrm{V}_{\mathrm{z}} \\
(\mathrm{ml} / \mathrm{kg})\end{array}$ \\
\hline \multirow{20}{*}{$\mathrm{AQ}$} & \multirow{5}{*}{ Male } & 001 & \multirow{3}{*}{$\mathrm{AQ}$} & 0.083 & 11.10 & 8.9 & 8.50 & 8.61 & 3029 & 39059 \\
\hline & & 002 & & 0.083 & 9.75 & 8.6 & 8.27 & 8.38 & 3144 & 38978 \\
\hline & & 003 & & 0.083 & 13.54 & 9.4 & 7.30 & 7.39 & 3333 & 45370 \\
\hline & & & Mean & 0.083 & 11.47 & 9.0 & 8.02 & 8.13 & 3169 & 41136 \\
\hline & & & SD & 0.000 & 1.92 & 0.4 & 0.63 & 0.65 & 153 & 3667 \\
\hline & \multirow{5}{*}{ Female } & $104 \dagger$ & \multirow{3}{*}{$\mathrm{AQ}$} & 0.0833 & 19.64 & 9.3 & 15.37 & 15.58 & 1668 & 22366 \\
\hline & & $005 t$ & & 0.0833 & 10.23 & n.a. & n.a. & n.a. & n.a. & n.a. \\
\hline & & $006+$ & & 0.0833 & 12.31 & n.a. & n.a. & n.a. & n.a. & n.a. \\
\hline & & & Mean & 0.083 & 14.06 & 9.3 & 15.37 & 15.58 & 1668 & 22366 \\
\hline & & & SD & 0.000 & 4.95 & n.c. & n.c. & n.c. & n.c. & n.c. \\
\hline & \multirow{5}{*}{ Male } & 001 & \multirow{3}{*}{ DEAQ } & 4.0 & 0.39 & 22.9 & 9.03 & 12.17 & n.c. & n.c. \\
\hline & & 002 & & 6.0 & 0.33 & 20.3 & 8.30 & 10.76 & n.c. & n.c. \\
\hline & & 003 & & 6.0 & 0.28 & 17.9 & 7.12 & 8.58 & n.c. & n.c. \\
\hline & & & Mean & 5.3 & 0.33 & 20.4 & 8.15 & 10.50 & n.c. & n.c. \\
\hline & & & SD & 1.2 & 0.06 & 2.5 & 0.96 & 1.81 & n.c. & n.c. \\
\hline & \multirow{5}{*}{ Female } & 104 & \multirow{3}{*}{ DEAQ } & 4.0 & 0.51 & 18.5 & 11.24 & 13.89 & n.c. & n.c. \\
\hline & & 005 & & n.a. & n.a. & n.a. & n.a. & n.a. & n.c. & n.c. \\
\hline & & 006 & & n.a. & n.a. & n.a. & n.a. & n.a. & n.c. & n.c. \\
\hline & & & Mean & 4.0 & 0.51 & 18.5 & 11.24 & 13.89 & n.c. & n.c. \\
\hline & & & SD & n.c. & n.c. & n.c. & n.c. & n.c. & n.c. & n.c. \\
\hline \multirow{10}{*}{ DEAQ } & \multirow{5}{*}{ Male } & 007 & \multirow{3}{*}{ DEAQ } & 0.083 & 7.29 & 12.1 & 20.69 & 21.73 & 1382 & 23280 \\
\hline & & 008 & & 0.083 & 7.87 & 11.3 & 22.31 & 23.17 & 1296 & 21175 \\
\hline & & 009 & & 0.083 & 7.41 & 14.8 & 24.59 & 26.86 & 1121 & 23939 \\
\hline & & & Mean & 0.083 & 7.53 & 12.7 & 22.53 & 23.92 & 1266 & 22798 \\
\hline & & & SD & 0.000 & 0.31 & 1.8 & 1.96 & 2.65 & 133 & 1444 \\
\hline & \multirow{5}{*}{ Female } & 010 & \multirow{3}{*}{ DEAQ } & 0.083 & 8.27 & 12.4 & 20.84 & 21.95 & 1366 & 24456 \\
\hline & & 011 & & 0.083 & 4.94 & 12.2 & 17.03 & 17.91 & 1681 & 29600 \\
\hline & & 012 & & 0.083 & 7.38 & 13.3 & 18.94 & 20.24 & 1478 & 28406 \\
\hline & & & Mean & 0.083 & 6.86 & 12.6 & 18.93 & 20.03 & 1508 & 27487 \\
\hline & & & SD & 0.000 & 1.72 & 0.6 & 1.91 & 2.03 & 160 & 2692 \\
\hline
\end{tabular}

Pharmacokinetics of individual male and female NZW rabbits were determined after

administered a single dose of $10 \mathrm{mg} / \mathrm{kg}$ of AQ or DEAQ intravenously. $\mathrm{C}_{\max }$ denotes maximal plasma concentration, $\mathrm{T}_{\max }$ time when $\mathrm{C}_{\max }$ is achieved, $\mathrm{t}_{1 / 2}$ plasma half-life, $\mathrm{AUC}_{\text {last }}$ area under the curve between first and last time points, $\mathrm{AUC}_{\text {inf }}$ area under the curve between first and last infinite time points, $\mathrm{Cl}$ clearance, and $\mathrm{V}_{\mathrm{z}}$ volume of distribution. Mean values and standard deviations for each pharmacokinetic parameter were calculated for male or female rabbits, if possible. 


\begin{tabular}{|c|c|c|c|c|c|c|c|c|}
\hline Drug & Sex & Animal & Analyte & $\begin{array}{l}T_{\max } \\
\text { (hr) }\end{array}$ & $\begin{array}{l}C_{\max } \\
(\mu \mathrm{M})\end{array}$ & $\begin{array}{c}\mathrm{t}_{1 / 2} \\
\text { (hr) }\end{array}$ & $\begin{array}{c}\text { AUClast } \\
(\mathbf{h r} \cdot \boldsymbol{\mu M})\end{array}$ & $\begin{array}{r}\text { AUCinf } \\
(\mathbf{h r} \cdot \mu \mathrm{M})\end{array}$ \\
\hline \multirow{20}{*}{$\mathrm{AQ}$} & \multirow{5}{*}{ Male } & 001 & \multirow{3}{*}{$\mathrm{AQ}$} & 1.00 & 0.85 & 1.9 & 2.46 & 2.66 \\
\hline & & 002 & & 2.00 & 0.99 & 3.9 & 5.31 & 5.39 \\
\hline & & 003 & & 1.00 & 0.56 & 3.4 & 2.79 & 3.60 \\
\hline & & & Mean & 1.00 & 0.80 & 3.1 & 3.52 & 3.88 \\
\hline & & & SD & 0.60 & 0.22 & 1.0 & 1.56 & 1.39 \\
\hline & \multirow{5}{*}{ Female } & 004 & \multirow{3}{*}{$\mathrm{AQ}$} & 2.00 & 0.89 & 1.9 & 3.10 & 3.39 \\
\hline & & 005 & & 2.00 & 1.08 & 4.5 & 5.11 & 5.22 \\
\hline & & 006 & & 1.00 & 1.22 & 4.6 & 5.83 & 5.93 \\
\hline & & & Mean & 2.00 & 1.07 & 3.7 & 4.68 & 4.84 \\
\hline & & & SD & 0.60 & 0.16 & 1.5 & 1.42 & 1.31 \\
\hline & \multirow{5}{*}{ Male } & 001 & \multirow{3}{*}{ DEAQ } & 1.00 & 1.86 & 12.4 & 20.03 & 21.56 \\
\hline & & 002 & & 2.00 & 2.90 & 11.9 & 35.49 & 38.38 \\
\hline & & 003 & & 2.00 & 1.39 & 9.6 & 22.68 & 23.57 \\
\hline & & & Mean & 2.00 & 2.05 & 11.3 & 26.07 & 27.84 \\
\hline & & & SD & 0.60 & 0.77 & 1.5 & 8.26 & 9.19 \\
\hline & \multirow{5}{*}{ Female } & 004 & \multirow{3}{*}{ DEAQ } & 2.00 & 3.42 & 10.9 & 28.23 & 29.88 \\
\hline & & 005 & & 2.00 & 2.56 & 11.3 & 32.27 & 34.29 \\
\hline & & 006 & & 1.00 & 3.14 & 9.4 & 39.18 & 40.48 \\
\hline & & & Mean & 2.00 & 3.04 & 10.5 & 33.23 & 34.88 \\
\hline & & & SD & 0.60 & 0.44 & 1.0 & 5.54 & 5.33 \\
\hline
\end{tabular}

Table S4: Pharmacokinetics of individual rabbits after oral administration of AQ.

Pharmacokinetics of individual male and female NZW rabbits were determined after administered a single dose of $10 \mathrm{mg} / \mathrm{kg}$ of $\mathrm{AQ}$ orally. $\mathrm{C}_{\max }$ denotes maximal plasma concentration, $\mathrm{T}_{\max }$ time when $\mathrm{C}_{\max }$ is achieved, $\mathrm{t}_{1 / 2}$ plasma half-life, $\mathrm{AUC}_{\text {last }}$ area under the curve between first and last time points, $\mathrm{AUC}_{\mathrm{inf}}$ area under the curve between first and last infinite time points, $\mathrm{Cl}$ clearance, and $\mathrm{V}_{\mathrm{z}}$ volume of distribution. Mean values and standard deviations for each pharmacokinetic parameter were calculated for male or female rabbits. 
Challenge

\begin{tabular}{|c|c|c|c|c|c|c|c|c|c|c|c|c|c|}
\hline Post-Challenge (days) & $n(\mathbf{M} / \mathbf{F})$ & -7 & $\mathbf{0}$ & $\mathbf{0}$ & 0.5 & 1.0 & 1.5 & 2.0 & 2.5 & 3 & 3.5 & 4 & 4.5 \\
\hline Post-Challenge (hours) & & -168 & 0 & 0.08 & 12 & 24 & 36 & 48 & 60 & 72 & 84 & 96 & 108 \\
\hline $\begin{array}{c}\mathrm{AQ}-5 \mathrm{mg} / \mathrm{kg}^{*} \\
\text { Group } 1\end{array}$ & $5(3 / 2)$ & $\mathrm{x}$ & - & $\mathrm{AQ}$ & $\mathrm{AQ}$ & $\begin{array}{c}\mathrm{AQ} \\
\mathrm{x}\end{array}$ & $\mathrm{AQ}$ & $\begin{array}{c}\mathrm{AQ} \\
\mathrm{x}\end{array}$ & $\mathrm{AQ}$ & $\mathrm{x}$ & - & - & - \\
\hline $\begin{array}{c}\mathrm{AQ}-20 \mathrm{mg} / \mathrm{kg}^{*} \\
\text { Group } 2\end{array}$ & $5(3 / 2)$ & $\mathrm{x}$ & - & AQ & $\mathrm{AQ}$ & $\begin{array}{c}\mathrm{AQ} \\
\mathrm{x}\end{array}$ & $\mathrm{AQ}$ & $\begin{array}{c}\mathrm{AQ} \\
\mathrm{x}\end{array}$ & $\mathrm{AQ}$ & $\mathrm{x}$ & - & - & - \\
\hline $\begin{array}{c}\text { Control - Untreated } \\
\text { Group } 3\end{array}$ & $6(3 / 3)$ & $\mathrm{x}$ & - & - & - & $\mathrm{x}$ & - & $\mathrm{x}$ & - & $\mathrm{x}$ & - & - & - \\
\hline $\begin{array}{c}\mathrm{AQ}-1.25 \mathrm{mg} / \mathrm{kg}^{*} \\
\text { Group } 4\end{array}$ & $5(2 / 3)$ & $\mathrm{x}$ & - & AQ & $\mathrm{AQ}$ & $\begin{array}{c}\mathrm{AQ} \\
\mathrm{x}\end{array}$ & $\mathrm{AQ}$ & $\begin{array}{c}\mathrm{AQ} \\
\mathrm{x}\end{array}$ & $\mathrm{AQ}$ & $\begin{array}{c}\mathrm{AQ} \\
\mathrm{x}\end{array}$ & $\mathrm{AQ}$ & $\mathrm{AQ}$ & $\mathrm{AQ}$ \\
\hline $\begin{array}{l}\mathrm{AQ}-5 \mathrm{mg} / \mathrm{kg}^{*} \\
\text { Group } 5\end{array}$ & $5(2 / 3)$ & $\mathrm{x}$ & - & $\mathrm{AQ}$ & $\mathrm{AQ}$ & $\begin{array}{c}\mathrm{AQ} \\
\mathrm{x}\end{array}$ & $\mathrm{AQ}$ & $\begin{array}{c}\mathrm{AQ} \\
\mathrm{x}\end{array}$ & $\mathrm{AQ}$ & $\begin{array}{c}\mathrm{AQ} \\
\mathrm{x}\end{array}$ & $\mathrm{AQ}$ & AQ & $\mathrm{AQ}$ \\
\hline
\end{tabular}

Table S5: Therapeutic dosing of AQ and blood collection schedule of anthrax-infected

rabbits. Anthrax-infected NZW white rabbits were administered varying doses of AQ for either 3 or 5 days at the time of aerosol exposure to $200 \mathrm{LD}_{50}$ of purified B. anthracis Ames spores. Blood samples of animals were collected prior to the anthrax challenge and once daily for three days post-challenge. 


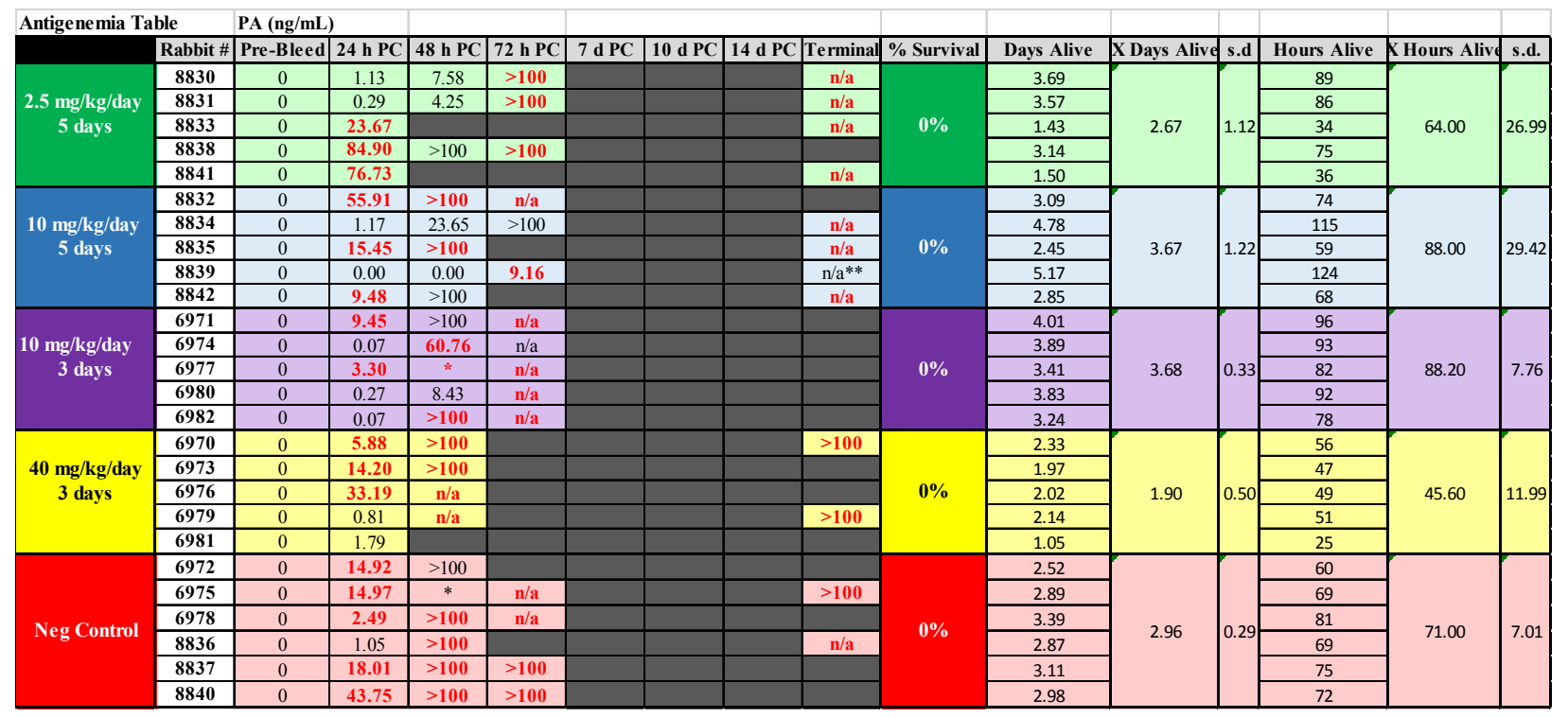

Table S6: Anthrax-related events of individual rabbits after oral administration of AQ.

Survival, bacteremia, and antigenemia (PA concentration in serum) of individual male and female NZW rabbits were determined in the absence or presence of various single doses of AQ. PC stands for Post Challenge. Numbers denote the PA antigen concentrations in blood at the designated times, while numbers in red font show the animals with bacteremia. * Not enough sample to assay. ** Terminal blood for 8839 was collected and cultured from a serum separator tube (SST), and not a Wampole tube. $\mathrm{n} / \mathrm{a}=$ Sample taken for bacteremia analysis, but not for antigenemia analysis. 


\begin{tabular}{|c|c|c|c|c|c|c|c|c|c|c|c|c|c|c|c|c|c|c|}
\hline & & & alle & & & & & & & & & & & & & & & \\
\hline Post-Challenge (days) & $n \dot{t}$ & -7 & $\mathbf{0}$ & $\mathbf{0}$ & 0.5 & 1.0 & 1.5 & 2.0 & 2.5 & 3 & 3.5 & 4 & 4.5 & 5 & 7 & 10 & 14 & 21 \\
\hline Post-Challenge (hours) & & -168 & 0 & 0.5 & 12 & 24 & 36 & 48 & 60 & 72 & 84 & 96 & 108 & 120 & 168 & 240 & 336 & 504 \\
\hline $\begin{array}{c}\mathrm{AQ}-5 \mathrm{mg} / \mathrm{kg} *+ \\
\text { Levofloxacin }-1.6 \mathrm{mg} / \mathrm{kg} \dagger\end{array}$ & 12 & $\mathrm{x}$ & - & AQ & $\mathrm{AQ}$ & $\begin{array}{c}\text { AQ } \\
+ \\
\text { Levo } \\
x\end{array}$ & AQ & $\begin{array}{c}\text { AQ } \\
+ \\
\text { Levo } \\
x\end{array}$ & $\mathrm{AQ}$ & $\begin{array}{c}\text { AQ } \\
+ \\
\text { Levo } \\
x\end{array}$ & AQ & $\begin{array}{c}\mathrm{AQ} \\
+ \\
\text { Levo } \\
\mathrm{x}\end{array}$ & $\mathrm{AQ}$ & $\begin{array}{c}\text { Levo } \\
x\end{array}$ & $\mathrm{x}$ & $\mathrm{x}$ & $\mathrm{x}$ & $\mathrm{x}$ \\
\hline Levofloxacin - $1.6 \mathrm{mg} / \mathrm{kg} \dagger$ & 12 & $\mathrm{x}$ & - & - & - & $\begin{array}{c}\text { Levo } \\
\mathrm{x}\end{array}$ & - & $\begin{array}{c}\text { Levo } \\
x\end{array}$ & - & $\begin{array}{c}\text { Levo } \\
\mathrm{x}\end{array}$ & - & $\begin{array}{c}\text { Levo } \\
\mathrm{x}\end{array}$ & - & $\begin{array}{c}\text { Levo } \\
\mathrm{x}\end{array}$ & $\mathrm{x}$ & $\mathrm{x}$ & $\mathrm{x}$ & $\mathrm{x}$ \\
\hline Control - Untreated & 4 & $\mathrm{x}$ & - & - & - & $\mathrm{x}$ & - & $\mathrm{x}$ & - & $\mathrm{x}$ & - & $\mathrm{x}$ & - & $\mathrm{x}$ & $\mathrm{x}$ & $\mathrm{x}$ & $\mathrm{x}$ & $\mathrm{x}$ \\
\hline
\end{tabular}

Table S7: Therapeutic dosing and blood collection schedule of anthrax-infected rabbits receiving levofloxacin and $\mathbf{A Q}$. Anthrax-infected NZW white rabbits were administered with a suboptimal dose $(1.6 \mathrm{mg} / \mathrm{kg} /$ day) of levofloxacin or with $10 \mathrm{mg} / \mathrm{kg} / \mathrm{day}$ of $\mathrm{AQ}$ in addition to levofloxacin. Blood samples of animals were collected prior to the anthrax challenge, once daily for five days post-challenge, and several times after seven days post-challenge if animals still had not succumbed to infection. 


\begin{tabular}{|c|c|c|c|c|c|c|c|c|c|c|c|c|c|c|c|}
\hline \multicolumn{2}{|c|}{ Serum $P A$ concentration } & $(\mathrm{ng} / \mathrm{ml})$ & & & & & & & & & & & & & \\
\hline & Rabbit \# & Pre-Bleed & 24 h PC & 48 h PC & 72 h PC & 96 h PC & 120 h PC & 7 days $\mathrm{PC}$ & 10 days $P C$ & 14 days $P C$ & 21 days $P C$ & \begin{tabular}{|l|} 
Terminal \\
\end{tabular} & $\%$ Survival & Days Alive & Hours Alive \\
\hline \multirow{12}{*}{ Levo Only } & 44820 & 0.00 & 33.16 & 34.71 & 14.13 & 151.14 & 674.92 & 59720.40 & & & & & \multirow{12}{*}{$33 \%$} & 6.98 & 167 \\
\hline & 44822 & 0.00 & 16.46 & 203.04 & \begin{tabular}{|l|}
273.02 \\
\end{tabular} & 962.15 & 5932.78 & & & & & & & 5.00 & 120 \\
\hline & 44825 & 0.00 & 3.44 & 18.04 & 43.71 & 42.78 & 72.86 & & & & & & & 4.89 & 117 \\
\hline & 44827 & 0.00 & 236.64 & 93.21 & \begin{tabular}{|l|}
150.27 \\
\end{tabular} & 1088.40 & 492.40 & 4.36 & 0.05 & & & & & 10.02 & 240 \\
\hline & 44830 & 0.00 & 4.52 & \begin{tabular}{|l|}
132.67 \\
\end{tabular} & \begin{tabular}{|l|}
1318.59 \\
\end{tabular} & & & & & & & + & & 4.02 & 96 \\
\hline & 44832 & 0.00 & 6.67 & 19.74 & 22.44 & 7.94 & 4.21 & 0.00 & 0.00 & 0.02 & 0.00 & $\mathrm{n} / \mathrm{a}$ & & 21.00 & 504 \\
\hline & 43552 & 0.00 & 2.96 & 22.27 & 105.64 & 481.10 & 3629.02 & & & & & + & & 5.89 & 141 \\
\hline & 43554 & 0.00 & 7.29 & 14.59 & 17.44 & 3.78 & 2.37 & 0.00 & 0.00 & 0.00 & 0.00 & $n / a$ & & 21.00 & 504 \\
\hline & 43556 & 0.00 & 0.95 & 7.48 & 3.00 & 4.08 & 1.17 & 76.94 & 0.00 & 0.04 & 0.00 & $n / a$ & & 21.00 & 504 \\
\hline & 43559 & 0.00 & 55.29 & 148.09 & 3100.38 & 8189.92 & & & & & & & & 4.00 & 96 \\
\hline & 43562 & 0.00 & 0.24 & 2.11 & 18.86 & 3.43 & 0.97 & 0.00 & 0.00 & 0.02 & 0.00 & $n / a$ & & 21.00 & 504 \\
\hline & 43564 & 0.00 & 3.48 & 28.49 & \begin{tabular}{|l|}
983.95 \\
\end{tabular} & 69404.46 & & & & & & & & 3.88 & 93 \\
\hline \multirow{12}{*}{ AQ \& Levo } & 44821 & 0.00 & 0.79 & 49.20 & 251.25 & 941.06 & 238653.02 & & & & & & \multirow{12}{*}{$67 \%$} & 5.02 & 121 \\
\hline & 44823 & 0.00 & 5.21 & 7.54 & ND & 100.24 & 295.18 & 0.00 & 0.00 & 0.00 & 0.00 & $n / a$ & & 21.00 & 504 \\
\hline & 44826 & 0.00 & 1.19 & 15.64 & 5.06 & 9.84 & 19.12 & 0.00 & 0.00 & 0.02 & 0.00 & $n / a$ & & 21.00 & 504 \\
\hline & 44828 & 0.00 & 40.47 & 17.75 & 4.93 & 3.03 & 0.91 & 0.00 & 0.00 & 0.00 & 0.00 & $n / a$ & & 21.00 & 504 \\
\hline & 44831 & 0.00 & 2.55 & 10.68 & 63.05 & 57.22 & 47.98 & 0.00 & 0.00 & 0.04 & 0.00 & $n / a$ & & 21.00 & 504 \\
\hline & 44833 & 0.00 & 0.11 & 6.42 & 28.06 & 99.46 & 168.95 & & & & & - & & 5.32 & 128 \\
\hline & 43553 & 0.00 & 4.44 & 51.74 & 361.46 & 444.39 & & & & & & - & & 4.79 & 115 \\
\hline & 43555 & 0.00 & 17.71 & 410.46 & & & & & & & & + & & 2.70 & 65 \\
\hline & 43557 & 0.00 & 0.81 & 5.14 & 3.20 & 1.70 & 0.63 & 0.00 & 0.00 & 0.00 & 0.00 & $n / a$ & & 21.00 & 504 \\
\hline & 43560 & 0.00 & 1.14 & 5.01 & 1.27 & 1.54 & 0.00 & 0.00 & 0.00 & 0.00 & 0.00 & $n / a$ & & 21.00 & 504 \\
\hline & 43563 & 0.00 & 0.00 & 3.70 & 21.22 & 58.02 & 5.81 & 0.17 & 0.00 & 0.00 & 0.00 & $\mathrm{n} / \mathrm{a}$ & & 21.00 & 504 \\
\hline & 43565 & 0.00 & 3.11 & 42.42 & 58.83 & 9.86 & 27.24 & 34.69 & 0.00 & 0.00 & 0.00 & $\mathrm{n} / \mathrm{a}$ & & 21.00 & 504 \\
\hline \multirow{4}{*}{ Neg. Control } & 44824 & 0.00 & 2.85 & 14.48 & 1492.90 & & & & & & & & \multirow{4}{*}{$0 \%$} & 2.91 & 70 \\
\hline & 44829 & 0.00 & 5.94 & 1887.30 & & & & & & & & & & 2.79 & 67 \\
\hline & 43558 & 0.00 & 0.34 & 1555.41 & & & & & & & & + & & 2.29 & 55 \\
\hline & 43561 & 0.00 & 42.56 & 828.31 & & & & & & & & + & & 2.51 & 60 \\
\hline
\end{tabular}

Table S8: Anthrax-related events of individual rabbits after oral administration of AQ.

Survival, bacteremia, and antigenemia (PA concentration in serum) of individual male and female NZW rabbits were determined in the absence or presence of Levofloxacin with and without 10 $\mathrm{mg} / \mathrm{kg} /$ day of AQ. Numbers denote the PA antigen concentrations in blood at the designated times, while numbers in red font show the animals with bacteremia. PC stands for Post Challenge. ND not determined for serum PA concentration. 


\begin{tabular}{|c|c|c|c|c|c|c|c|c|c|c|c|c|c|c|c|c|c|c|c|c|}
\hline \multicolumn{21}{|c|}{ Challenge } \\
\hline Post-Challenge (days) & $n+$ & -7 & $\mathbf{0}$ & 0 & 0.5 & 1.0 & 1.5 & 2.0 & 2.5 & 3 & 3.5 & 4 & 4.5 & 5 & 5.5 & 6 & 6.5 & 7 & 10 & 14 \\
\hline Post-Challenge (hours) & & -168 & 0 & 0.5 & 12 & 24 & 36 & 48 & 60 & 72 & 84 & 96 & 108 & 120 & 132 & 144 & 156 & 168 & 240 & 336 \\
\hline $\begin{array}{c}\mathrm{AQ}(0.5 \mathrm{~h})-5 \mathrm{mg} / \mathrm{kg}^{*}+ \\
\text { Levofloxacin }-1.6 \mathrm{mg} / \mathrm{kg} \dagger\end{array}$ & 12 & $x \S$ & - & $\mathrm{AQ}$ & $\begin{array}{c}\mathrm{AQ} \\
\mathrm{x}\end{array}$ & $\begin{array}{c}\text { AQ } \\
+ \\
\text { Levo } \\
x\end{array}$ & $\mathrm{AQ}$ & $\begin{array}{c}\text { AQ } \\
+ \\
\text { Levo } \\
x\end{array}$ & AQ & $\begin{array}{c}\text { AQ } \\
+ \\
\text { Levo } \\
x \S\end{array}$ & $\mathrm{AQ}$ & $\begin{array}{c}\text { AQ } \\
+ \\
\text { Levo } \\
x\end{array}$ & $\mathrm{AQ}$ & $\begin{array}{c}\text { Levo } \\
\mathrm{x}\end{array}$ & - & - & - & $\mathrm{x} \S$ & $x \S$ & $\mathrm{x} \S$ \\
\hline $\begin{array}{c}\mathrm{AQ}(12 \mathrm{~h})-5 \mathrm{mg} / \mathrm{kg}^{*}+ \\
\text { Levofloxacin }-1.6 \mathrm{mg} / \mathrm{kg} \dagger\end{array}$ & 12 & $\mathrm{x} \S$ & - & - & $\begin{array}{c}\mathrm{AQ} \\
\mathrm{x}\end{array}$ & $\begin{array}{c}\text { AQ } \\
+ \\
\text { Levo } \\
x\end{array}$ & $\mathrm{AQ}$ & $\begin{array}{c}\text { AQ } \\
+ \\
\text { Levo } \\
x\end{array}$ & $\mathrm{AQ}$ & $\begin{array}{c}\mathrm{AQ} \\
+ \\
\text { Levo } \\
\mathrm{x} \S\end{array}$ & $\mathrm{AQ}$ & $\begin{array}{c}\text { AQ } \\
+ \\
\text { Levo } \\
x\end{array}$ & $\mathrm{AQ}$ & $\begin{array}{c}\mathrm{AQ} \\
+ \\
\text { Levo } \\
\mathrm{x}\end{array}$ & - & - & - & $\mathrm{x} \S$ & $\mathrm{x} \S$ & $x \S$ \\
\hline $\begin{array}{c}\mathrm{AQ}(24 \mathrm{~h})-5 \mathrm{mg} / \mathrm{kg}^{*}+ \\
\text { Levofloxacin }-1.6 \mathrm{mg} / \mathrm{kg} \dagger\end{array}$ & 12 & $x \S$ & - & - & $\mathrm{x}$ & $\begin{array}{c}\text { AQ } \\
+ \\
\text { Levo } \\
x\end{array}$ & $\mathrm{AQ}$ & $\begin{array}{c}\text { AQ } \\
+ \\
\text { Levo } \\
x\end{array}$ & AQ & $\begin{array}{c}\mathrm{AQ} \\
+ \\
\text { Levo } \\
\mathrm{x} \S\end{array}$ & AQ & $\begin{array}{c}\text { AQ } \\
+ \\
\text { Levo } \\
x\end{array}$ & $\mathrm{AQ}$ & $\begin{array}{c}\mathrm{AQ} \\
+ \\
\text { Levo } \\
\mathrm{x}\end{array}$ & AQ & - & - & $\mathrm{x} \S$ & $x \S$ & $x \S$ \\
\hline $\begin{array}{c}\mathrm{AQ}(48 \mathrm{~h})-5 \mathrm{mg} / \mathrm{kg}^{*}+ \\
\text { Levofloxacin }-1.6 \mathrm{mg} / \mathrm{kg} \dagger\end{array}$ & 12 & $x \S$ & - & - & $\mathrm{x}$ & $\begin{array}{l}\text { Levo } \\
\mathrm{x}\end{array}$ & - & $\begin{array}{c}\text { AQ } \\
+ \\
\text { Levo } \\
x\end{array}$ & $\mathrm{AQ}$ & $\begin{array}{c}\text { AQ } \\
+ \\
\text { Levo } \\
x \S\end{array}$ & $\mathrm{AQ}$ & $\begin{array}{c}\text { AQ } \\
+ \\
\text { Levo } \\
x\end{array}$ & $\mathrm{AQ}$ & $\begin{array}{c}\mathrm{AQ} \\
+ \\
\text { Levo } \\
\mathrm{x}\end{array}$ & AQ & AQ & $\mathrm{AQ}$ & $\mathrm{x} \S$ & $\mathrm{x} \S$ & $\mathrm{x} \S$ \\
\hline Levofloxacin $-1.6 \mathrm{mg} / \mathrm{kg} \dagger$ & 4 & $\mathrm{x} \S$ & - & - & $\mathrm{x}$ & $\begin{array}{c}\text { Levo } \\
\mathrm{x}\end{array}$ & - & $\begin{array}{c}\text { Levo } \\
\mathrm{x}\end{array}$ & - & $\begin{array}{c}\text { Levo } \\
x \S\end{array}$ & - & $\begin{array}{c}\text { Levo } \\
\mathrm{x}\end{array}$ & - & $\begin{array}{c}\text { Levo } \\
\mathrm{x}\end{array}$ & - & - & - & $x \S$ & $\mathrm{x} \S$ & $x \S$ \\
\hline Control - Untreated & 4 & $x \S$ & - & - & $\mathrm{x}$ & $\mathrm{x}$ & - & $\mathrm{x}$ & - & $\mathrm{x} \S$ & - & $\mathrm{x}$ & - & $\mathrm{x}$ & - & - & - & $x \S$ & $\mathrm{x} \S$ & $x \S$ \\
\hline
\end{tabular}

Table S9: Therapeutic dosing and blood collection schedule of anthrax-infected rabbits receiving levofloxacin and delayed AQ treatment. Anthrax-infected NZW white rabbits were administered with a suboptimal dose $(1.6 \mathrm{mg} / \mathrm{kg} /$ day $)$ of levofloxacin or with $10 \mathrm{mg} / \mathrm{kg} /$ day of AQ in addition to levofloxacin at 30 minutes, 12 hours, 24 hours, or 48 hours post-infection. All treatments were given for a total of five days from start time. Blood samples of animals were collected prior to the anthrax challenge, at least once daily for five days post-challenge, and several times after seven days post-challenge if animals still had not succumbed to infection. Assessment for anti-PA IgG was conducted for all animals prior to challenge, as well as 3-, 7-, 10-, and 14days post anthrax challenge. 


\begin{tabular}{|c|c|c|c|}
\hline Side Effect & \# Articles & Side Effect & \# Articles \\
\hline Agranulocytosis & 13 & Oedema & 3 \\
\hline Jaundice & 7 & Pruritus & 3 \\
\hline Nausea & 7 & Abdominal discomfort & 2 \\
\hline Vomiting & 7 & Ascites & 2 \\
\hline Hepatitis & 6 & Asterixis & 2 \\
\hline Headache & 5 & Atrophy & 2 \\
\hline Malaise & 5 & Blindness & 2 \\
\hline Encephalopathy & 4 & Chills & 2 \\
\hline Hepatic encephalopathy & 4 & Cholestasis & 2 \\
\hline Necrosis & 4 & Corneal deposits & 2 \\
\hline Rash & 4 & Diarrhea & 2 \\
\hline Abdominal pain & 3 & Erythema & 2 \\
\hline Anorexia & 3 & Gastroenteritis & 2 \\
\hline Asthenia & 3 & Hepatotoxicity & 2 \\
\hline Fatigue & 3 & Herpes simplex & 2 \\
\hline Fibrosis & 3 & Mucosal pigmentation & 2 \\
\hline Hepatitis a & 3 & Renal failure & 2 \\
\hline Hepatitis b & 3 & Sepsis & 2 \\
\hline Inflammation & 3 & Skin reaction & 2 \\
\hline Lethargy & 3 & Thrombocytopenia & 2 \\
\hline Melanosis & 3 & Ulcer & 2 \\
\hline Neutropenia & 3 & & \\
\hline & & & \\
\hline
\end{tabular}

Table S10: Identification of side effects in AQ safety articles via Python. A list of general 7,058 side-effects, which excluded the side-effect "malaria", was utilized. The number of articles that mention a side effect from the list is counted in the set of 25 AQ safety articles extracted from the manual systemic literature review. Python Parser Library was used to search words and phrases in the main body of each article (excluding references). Only side effects mentioned in more than one article were included in this Table. 


\begin{tabular}{|c|c|c|c|}
\hline Side Effect & \# Articles & Side Effect & \# Articles \\
\hline Agranulocytosis & 13 & Asthenia & 3 \\
\hline Jaundice & 9 & Atrophy & 3 \\
\hline Nausea & 8 & Cytopenia & 3 \\
\hline Headache & 7 & Fatigue & 3 \\
\hline Vomiting & 7 & Fibrosis & 3 \\
\hline Hepatitis & 6 & Hepatitis a & 3 \\
\hline Malaise & 5 & Hepatitis b & 3 \\
\hline Encephalopathy & 4 & Inflammation & 3 \\
\hline Melanosis & 4 & Lethargy & 3 \\
\hline Necrosis & 4 & Oedema & 3 \\
\hline Neutropenia & 4 & Rash & 3 \\
\hline Pruritus & 4 & Skin reaction & 3 \\
\hline Abdominal pain & 3 & Visual disturbance & 3 \\
\hline Anorexia & 3 & & \\
\hline
\end{tabular}

Table S11: Identification of side effects in AQ safety articles via Matlab. A list of general 7,058 side-effects, which excluded the side-effect "malaria", was utilized. The number of articles that mention a side effect from the list is counted in the set of 25 AQ safety articles extracted from the manual systemic literature review. Matlab Text Analytics Toolbox was used to search words and phrases in the main body of each article (excluding references). Only side effects mentioned in more than two articles were included in this Table. 


\begin{tabular}{|c|c|c|c|}
\hline Side Effect & \# Articles & Side Effect & \# Articles \\
\hline Vomiting & 462 & Lethargy & 52 \\
\hline Agranulocytosis & 233 & Malaise & 51 \\
\hline Headache & 223 & Plasmodium falciparum infection & 51 \\
\hline Nausea & 199 & Inflammation & 50 \\
\hline Hepatitis & 176 & Renal failure & 43 \\
\hline Diarrhea & 174 & Meningitis & 38 \\
\hline Abdominal pain & 166 & Liver injury & 37 \\
\hline Anorexia & 131 & Erythema & 36 \\
\hline Pruritus & 131 & Oedema & 36 \\
\hline Hepatotoxicity & 127 & Abdominal discomfort & 30 \\
\hline Hypersensitivity & 123 & Dermatitis & 30 \\
\hline Rash & 109 & Leprosy & 29 \\
\hline Tuberculosis & 104 & Influenza & 27 \\
\hline Neutropenia & 97 & Hepatitis b & 26 \\
\hline Jaundice & 89 & Thrombocytopenia & 25 \\
\hline Fatigue & 79 & Genetic polymorphism & 24 \\
\hline Necrosis & 68 & Aspiration & 23 \\
\hline Coma & 64 & Phagocytosis & 22 \\
\hline Arthritis & 61 & Fasting & 21 \\
\hline Chills & 59 & Gastroenteritis & 21 \\
\hline Splenomegaly & 59 & Rigors & 21 \\
\hline Asthenia & 52 & & \\
\hline
\end{tabular}

Table S12: Identification of side effects in AQ articles via Python. A list of 148 side-effects previously found in the set of $25 \mathrm{AQ}$ safety articles extracted from the manual systemic literature review was utilized. The number of articles that mention a side effect from this selected list is counted in a large publication database of all available AQ papers (1,807 articles in pdf format). Python Parser Library was used to search words and phrases in the main body of each article (excluding references). Only side effects mentioned in more than 20 articles were included in this Table. 


\begin{tabular}{|c|c|c|c|}
\hline Side Effect & \# Articles & Side Effect & \# Articles \\
\hline Vomiting & 130 & Jaundice & 33 \\
\hline Anemia & 68 & Pneumonia & 29 \\
\hline Nausea & 60 & Plasmodium falciparum infection & 24 \\
\hline Headache & 59 & Anorexia & 23 \\
\hline Agranulocytosis & 55 & Splenomegaly & 22 \\
\hline Diarrhea & 47 & Dizziness & 20 \\
\hline Hepatitis & 44 & Malaise & 20 \\
\hline Convulsion & 38 & Neutropenia & 20 \\
\hline Pruritus & 38 & Rash & 19 \\
\hline Malnutrition & 36 & Hypersensitivity & 18 \\
\hline Abdominal pain & 33 & & \\
\hline
\end{tabular}

Table S13: Identification of side effects in AQ articles via Matlab. A list of general 7,058 sideeffects, which excluded the side-effect "malaria", was utilized. The number of articles that mention a side effect from the list is counted in the set of 411 AQ articles extracted from the manual systemic literature review. Matlab Text Analytics Toolbox was used to search words and phrases in the main body of each article (excluding references). 